\title{
Research on Fuzzy-Logic Algorithm of Intelligent Generating Test Paper
}

\author{
http://dx.doi.org/10.3991/ijet.v8i4.2906 \\ Qing-yun RU, Jing-yi DU \\ Henan Mechanic and Electric Engineering College, Xinxiang, China
}

\begin{abstract}
Intelligent generating test paper can be thought of as a typical fuzzy mathematical logic problem. First, the paper analyzes the quantitative evaluation criteria of the traditional test paper generation questions and shows the existing problems in practical application of it. Then the paper introduces two different fuzzy constraints principal disjunctive normal form concept and proposes an evaluation system combining quantitative with qualitative. Viewing at the particularity of constraint satisfaction problems of intelligent generating test paper, the paper uses heuristic knowledge and applies constraint satisfaction method combining generation test with membership correction constraint satisfaction methods to realize intelligent generating test paper. The experiment shows that this method obtains good results.
\end{abstract}

Index Terms-Conjunction, Disjunction, fuzzy constraint paradigm, Intelligent Generating Test Paper, membership correction algorithm

\section{INTRODUCTION}

With the deepening of the teaching reform, CAI technology develops rapidly and the test database of various disciplines have been brought forth. The test database controls teaching quality from macro and it takes an important role in macro-guidance for construction and reform of the curriculum. It can provide multi-angle, multi-level, multi-functional services for teaching management, testing and evaluation etc. Generating test paper is an important basic function of question database system. Intelligent test paper generation focuses on the knowledge of experts in education and makes use of artificial intelligence technology to automatically select the questions from the question database to form the papers meeting various performance requirements. The distinctive feature of these papers is objectivity, accuracy and reliability for the teaching evaluation.

According to the principles of education measurement and education statistics, traditionally, difficulty, discrimination, reliability and validity are all thought of as the main properties of paper. However, because it builds on the basis of measurement and statistics, to achieve the quantitative evaluation objectively, there is a big deviation between the result and the actual situation [1]. More realistic approach is based on the experience of education experts to construct a more practical and more effective evaluation system combining qualitative with quantitative.

Papers should meet the appropriate performance requirements, so the intelligent generating test paper is a typical constraint satisfaction problem (CSP) solution process. The solving of CSP problem usually makes use of standard backtracking algorithm [2]. For the intelligent test paper generation, if only using of standard backtracking algorithm, with the "strong constraint ", it often occurs that the formed papers are unable to meet the performance requirements. Since CSP is usually an NP problem, a variety of strategies and heuristic knowledge must be used. Therefore, based on the standard backtracking, this algorithm uses the logic main disjunction and membership local correction method to correct it, and effectively uses a variety of heuristic knowledge to obtain good results.

The intelligent generating test paper method proposed by the paper is used in the intelligent generating test paper system of computer assembly and maintenance test paper database, and the result is good.

II. PERformance EVAluation OF Qualitative AND QUANTITATIVE OF INTELLIGENT TEST PAPER GENERATION

\section{A. The Traditional Paper Evaluation Criteria}

In traditional evaluation of the papers, the difficulty, distinguish, of validity and reliability of test papers are thought of as standard.

The difficulty of test papers will be defined as:

$$
p=\frac{1}{w} \sum_{i=1}^{M} p_{i} W_{i}=1-\frac{\bar{X}}{W}
$$

In this formula, w is the full mark value of the papers, $\bar{X}$ is the full mark mean value of the examinees, $\mathrm{M}$ is the subject numbers of papers, $\mathrm{Pi}$ and $\mathrm{Wi}$ are the difficulty and score of $\mathrm{i}$ paper. The difficulty $\mathrm{P}$ determines the mean value of candidates. However, the difficulty value $\mathrm{Pi}$ of examination questions is difficult to be determined accurately. Because W needs a large number of statistical measurement (which is difficult to achieve), and it can not express the actual level of "teaching" and "learning" either. Therefore, the average mark determined by the paper difficulty $\mathrm{P}$ is often not consistent with the actual test results [3].

The discrimination of paper is also based on the discrimination of questions. It is defined as:

$$
D=\frac{1}{w} \sum_{i=1}^{M} D_{i} W_{i}=\alpha \delta+b
$$


In Equation (2), $\mathrm{a}$ and $\mathrm{b}$ are all constants. This expression shows that there exists linear relation between the discrimination $\mathrm{D}$ and the standard deviation $\delta$ of the paper, that is, the discrimination $\mathrm{D}$ of paper determines the standard deviation of paper and the discrimination D of questions is difficult to accurately determine. So D and $\delta$ naturally do not conform to reality.

Paper reliability refers to the reliability of the examination of the paper; and the reliability of test scores from the indicators is the levels of examination results, which reflects the true extent of candidate evaluation, in fact, the reliability test. It is defined as:

$$
\gamma_{\alpha}=\frac{S r^{2}}{S^{2}}=1-\frac{S e^{2}}{S^{2}} .
$$

Validity of examination paper refers to the index of examination mark performance after examining and it is the evaluation of examination results, which reflects the real level of examinees. It is validity of examination and is defined as:

$$
\gamma_{\alpha}=\frac{S r^{2}}{S^{2}}=\left(1-\frac{S e^{2}}{S^{2}}\right)-\frac{S e^{2}}{S^{2}} .
$$

$S r_{v}{ }^{2}$ is the variance of true and effective score, $S e_{e}{ }^{2}$ is the variance of the true and valid score, $\mathrm{Sr}^{2}=\mathrm{Sr}_{e}{ }^{2}$ ${ }_{+} \mathrm{Sr}_{v}{ }^{2}$. Obviously, the validity of the above formula is also a theory, not the actual calculations.

\section{B. Performance Evaluation of qualitative and quantitative about Intelligent Generating Test Paper}

The above condition shows that the traditional performance evaluation of the papers all build on the basis of property values questions. However, not only statistical measurement but also expertise experience is difficult to determine the exact quantitative attributes value of the questions. Therefore, quantitative evaluation of examination papers on a basis of this method is obviously not practical. It will be more practical using the method combining with qualitative and quantitative to determine the property value of the questions and establishing the evaluation criteria for the corresponding paper. Test scores and estimated time uses a quantitative expression and the section knowledge points of questions and the question type use the exact symbol amount to express. The rest properties including item difficulty, ability level, and teaching requirements degree can be expressed by fuzzy qualitative expression. The partial property and the property values can be shown in Table 1 .

TABLE I.

PARTIAL PRoperty of EXAMINATION AND MEMBERShiP VALUE

\begin{tabular}{|l|l|l|l|l|}
\hline Properties & \multicolumn{1}{|c|}{ Examination Type } & \multicolumn{1}{|c|}{ Item difficulty } & \multicolumn{1}{|c|}{ Ability Level } & Teaching Requirements \\
\hline \multirow{5}{*}{ Constraint } & Choice & Easy & Memorization & General \\
\cline { 2 - 5 } & Fill in the blanks & Middle & $\begin{array}{l}\text { Understand and } \\
\text { Application }\end{array}$ & Generally Main Points \\
\cline { 2 - 5 } & Problems & Difficulty & Analysis and Synthesis & Main Points \\
\cline { 2 - 5 } & Others & Hard & Research & Exceeding Requirements \\
\hline
\end{tabular}

According to the examination attributes, constraints of intelligent generating test paper can be divided into four categories: strong constraint, sub-constraint, flexible constraint and weak constraint. Strong constraint can be defined as a constraint must be satisfied, such as total scores of papers and the chapter knowledge points of papers and so on. Sub-constraint is defined as the priority constraints except for strong constraints such as the distribution of scores, questions number and questions type within each chapter knowledge of paper. These constraints are closely related to the reliability and validity of the papers. In accordance with Spears - Brown formula, the more the questions are, the more widely the knowledge point distributes and the more reasonable the reliability is. High reliability degree is a necessary condition for high validity.

Flexible constraint is defined as a constraint which allows a bound variable quantity to change in some certain range. Weak constraint is actually a reference constraint, such as the estimated time. The time using by each item is an inaccurate estimated value. So the estimated time is only used as reference, and the full marks of papers can partly reflect its binding actually.

\section{CONSTRAint SATISFACTION METHOD OF INTELLIGENT GENERATING TEST PAPER}

\section{A. Constraint}

To solve finite constraint satisfaction problem, the most simple and direct method is to generate the test method. This approach is clearly inefficient. In order to generate test method, backtracking is an improved method. It is a fixed instantiation order of variables, when a new variable is inconsistent with the previously assigned variables, tries another value of the variable domain until all the domain values are exhausted. [4] Intelligent Test Paper presents the search algorithm. Sequence is actually backtrackingtree-search-based method for solving the structure. Because CSP is a NP problem, which is usually the problem, the method failed to make full use of heuristic knowledge, not only on search efficiency, but also in the "strong binding " cases, are often not effective solutions.

Therefore, we bound test paper into the issue "strong constraint, "' Asia constraints ","soft constraints "and " weak constraint "and different levels, in the generated test method based on the use of heuristic knowledge, to take "fuzzy logic Local Amendment Act "to solve it. 
Strong constraint: Paper covers the knowledge points (total membership is 1) and Questions membership (percentage), which get their conjunction.

Asia constraints: distribution of questions in each chapter membership (ratio), examination papers and questions the objectivity of the non-repetitive, seeking their disjunction.

Flexible constraints: the proportion of questions of various difficulty, the proportion of questions of various ability levels, test requirements of the various teaching degree ratio.

Weak constraint: when the papers is with the estimates.

\section{B. Intelligent Algorithm for the Initial Papers}

The initial focus of papers consider the strong constraints and sub-constraint satisfaction problem, concrete steps are:

1) First, extract from the item bank all the questions to meet the requirements of papers covering of knowledge points as candidate questions;

2) Calculate the average of various kinds of the candidate questions;

3) Bound by papers and various kinds of questions out of proportion to the questions the subject of the number of each type of questions;

4) Questions in each chapter by the weight distribution of the knowledge points were calculated for each subject of the number of item types;

5)in each chapter of the subject item, the candidate item types is in accordance with the corresponding number of randomly selected questions not recently been selected to generate the initial papers.

\section{Intelligent Test Paper Local Amendments Act of Membership}

The basic algorithm of intelligence test paper is to generate a test method. The test papers generated by the type of constraints were tested. Strong constraints must be completely satisfied: for sub-base to meet the constraint should be: for flexible constraint satisfaction on a weighted evaluation function of the test; for the weak constraint given the test results only, not to meet the level of evaluation.

Since the initial papers have at least a strong base to meet the constraints and sub-constraints. Therefore, the initial papers to perfect addition to the main task are to further consider the papers out of the constraints, an important consideration for flexible constraint satisfaction. The weighted evaluation function $\mathrm{J}$ is defined as follows:

$$
J=\sum_{i=1}^{4} C^{i} \sum_{i=1}^{m_{i}}\left(\frac{\sum_{j=1}^{n_{l}^{i}} w_{l j}^{i}}{\sum_{k=1}^{n} W_{k}}-p_{l}^{i}\right) .
$$

Wk papers which said the theme of the first $k$ points, $n$ is the number of papers in the general questions for the class attribute of the first i-l j-attribute value types question marks, the property value and the corresponding item number, the property Value constraint value for the evaluation of the property to satisfy the weighting coefficient, $i=1,2,3,4$, respectively, for the kinds of questions that item attribute, the difficulty, ability level, degree of teaching requirements. When the evaluation function is equal to or less than a given value, the definition of flexibility meet all constraints of the paper.

\section{Intelligent Test Paper Main DNF}

For the sake of simplicity, assume A, B, C, D are the four kinds of questions, to choose two kinds of questions, the following three conditions, there are several election law. How to choose? (1) If the option A is C and D, to choose a type; (2) B and $\mathrm{C}$ can not have elections; (3) choose $\mathrm{C}$, you can not choose $\mathrm{D}$. The answer is: $(\mathrm{A} \rightarrow \mathrm{C}$ $\vee \mathrm{D}) \Lambda(\neg(\mathrm{B} \Lambda \mathrm{C})) \Lambda(\mathrm{C} \rightarrow \neg \mathrm{D})=\Sigma 0,1,2,4,5,9, \mathrm{~A}, \mathrm{D}$, and then be drawn according to the actual paradigm of discussion $(\neg \mathrm{A} \Lambda \mathrm{B} \Lambda \neg \mathrm{C} \Lambda \mathrm{D}) \vee(\mathrm{A} \Lambda \neg \mathrm{B} \Lambda \neg \mathrm{C} \Lambda \mathrm{D}) \vee$ $(\mathrm{A} \Lambda \neg \mathrm{B} \Lambda \mathrm{C} \Lambda \neg \mathrm{D})$.

Therefore, there are three election law, any kind can be taken.

\section{INTELLIGENT GROUPING EXAMPLE}

According to the above method of an intelligent test, we have succeeded designing an intelligent test item bank system which is composed of only a college paper discrete mathematics to illustrate the effectiveness of the method, in Henan mechanic and electric engineering college.

total score of 100 , estimated by the time 120 minutes

Chapter membership and knowledge point distribution shown in Table 2 (no special requirements for knowledge points)

(3) The kinds of questions, difficulty, ability levels, and teaching requirements of the degree of constraint can be shown in Table 3.

Accordance with the above constraints, intelligent test system of a component meets the requirements of the three papers A, B, C Vol. A paper form parameters which are listed in Table 2 and Table 4. The total score of 100 points, the time for questions is 120 minutes. 
TABLE II.

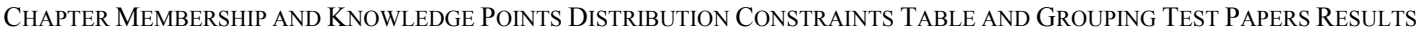

\begin{tabular}{|l|l|l|l|l|l|}
\hline Chapter & \multicolumn{1}{|c|}{$\begin{array}{c}\text { Object } \\
\text { Membership }\end{array}$} & \multicolumn{1}{c|}{$\begin{array}{c}\text { Actual } \\
\text { Membership }\end{array}$} & $\begin{array}{c}\text { Object } \\
\text { Membership }\end{array}$ & $\begin{array}{c}\text { Actual } \\
\text { Membership }\end{array}$ \\
\hline 1 & 0.2 & 0.19 & 5 & 0.1 & 0.9 \\
\hline 2 & 0.2 & 0.9 & 6 & 0.1 & 0.11 \\
\hline 3 & 0.1 & 0.23 & 7 & 0.05 & 0.6 \\
\hline 4 & 0.25 & 0.23 & & & \\
\hline
\end{tabular}

TABLE III.

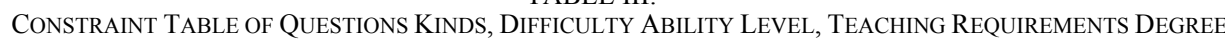

\begin{tabular}{|l|l|l|l|}
\hline \multicolumn{1}{|c|}{ Questions Kinds } & \multicolumn{1}{|c|}{ Difficulty } & \multicolumn{1}{c|}{ Ability Level } & \multicolumn{1}{c|}{ Teaching Requirements } \\
\hline Choice 0.3 & Easy 0.2 & Memorization 0.2 & Generally 0.2 \\
\hline Fill in blank 0.3 & Middle 0.4 & $\begin{array}{l}\text { Understand and Application } \\
0.4\end{array}$ & Generally Main Points0.4 \\
\hline Others & & Problems 0 & Exceeding Requirements 0 \\
\hline
\end{tabular}

TABLE IV.

Grouping Test PAPER Results TABle of Questions Kinds, DifFiculty ABILITy LEVELS AND TEACHING REQUIREMENTS

\begin{tabular}{|l|l|l|l|}
\hline \multicolumn{1}{|c|}{ Questions Kinds } & \multicolumn{1}{|c|}{ Difficulty } & \multicolumn{1}{c|}{ Ability Level } & \multicolumn{1}{c|}{ Teaching Requirements } \\
\hline Choice 0.31 & Easy 0.2 & Memorization 0.2 & Generally 0.21 \\
\hline Fill in blank 0.28 & Middle 0.45 & $\begin{array}{l}\text { Understand and Application } \\
0.41\end{array}$ & Generally Main Points 0.4 \\
\hline Others 0 & & Problems 0 & Exceeding Requirements 0 \\
\hline
\end{tabular}

The results can be seen from the test paper consisting of intelligent test system of examination papers to better meet the requirements of different types of constraints that the proposed intelligent test paper method.

\section{CONCLUSION}

This paper describes intelligent test paper method of discrete mathematics principal disjunctive, effectively increased flexibility and scientific of test paper, is a kind of good method.

\section{REFERENCES}

[1] Zhu Xu, Implement and Research of Math Question Bank System. Teaching and Teaching Materials, Vol. 2, 1995: pp.29-30.

[2] QU Xiao-tang, Research of the Intelligent Test Paper Composition Based of the Genetic Algorithm. Computer Knowledge and Technology(Academic Exchange), Vol.18, 2007: pp.5-9.

[3] KENNEDY J, EBERHART R, "A discrete binary version of the particle swarm algorithm", Proceedings of the World Multiconference on Systemic Cybernetics and Informatics, Orlando, USA. NJ: Piscatawary, 1997: pp. 4104-4108.

[4] Ming Zhu, Junpu Wang, ,Jingjie Wang, The general test library system, Computer Engineering, 1996: pp. 451-460.

[5] Gu. J, Efficient Local Search for Very Large-scale Satisfiability Problems, Sigart Bulletin, 1992: pp.8-12.
[6] David W Coit, Alice E Smith, David M Tate, Adaptive Penalty Methods for Genetic Optimization of Constrained Combinatorial Problems, NFORMS Journal on Computing, 1996: pp.172-183.

[7] Sun Jianyong, Shen Jianzhong, $\mathrm{Xu}$ Zongben, New Adaptive Genetic Algorithm, Journal of Xi'an Jiaotong University, 2000: pp.84-88.

[8] Holland J.H., Adaptation in Nature and Artificial System, The University of Michigan Press, 1998.

[9] Goldberg DE, Genetic Algorithms in Search, Optimization and Machine Learning, MA, Addison-Wesley Publishing, 1989.

[10] D.B. Fogel, Evolutionary, New York: IEEE Press. 1995.

\section{AUTHORS}

Qing-yun RU is with the Department of Computer Science and Technology, Henan Mechanic and Electric Engineering College, Xinxiang, CO 453002 China (email: ruqingyun@126.com).

Jing-yi DU is with the Department of Computer Science and Technology, Henan Mechanic and Electric Engineering College, Xinxiang, CO 453002 China (email: lovelypine@126.com).

Manuscript received 8 May 2013. Published as re-submitted by the authors 8 August 2013. 